\title{
Investigation of the Stability of a Test Particle in the Vicinity of Collinear Points with the Additional Influence of an Oblate Primary and a Triaxial-Stellar Companion in the Frame of ER3BP
}

\author{
Aminu Abubakar Hussain ${ }^{a}$, Aishetu Umar ${ }^{b}$ and Jagadish Singh ${ }^{c}$ \\ Department of Mathematics, Faculty of Science, Ahmadu Bello University Zaria, Nigeria. \\ adadinduniya@gmail.com, bumaraishetu33@yahoo.com, cjgds2004@yahoo.com
}

Keywords: Celestial mechanics, Collinear points, ER3BP.

\begin{abstract}
We investigate in the elliptic framework of the restricted three-body problem, the motion around the collinear libration points of an infinitesimal particle in the vicinity of an oblate primary and a triaxial stellar companion. The locations of the collinear libration points are affected by the eccentricity of the orbits, oblateness of the primary body and the triaxiality and luminosity of the secondary. A numerical analysis of the effects of the parameters on the positions of collinear libration points of CEN X-4 and PSR J1903+0327 reveals a general shift away from the smaller primary with increase in eccentricity and triaxiality factors and a shift towards the smaller primary with increase in the semi-major axis and oblateness of the primary on $L_{1}$. The collinear libration points remain unstable in spite of the introduction of these parameters.
\end{abstract}

\section{Introduction}

In Celestial mechanics and space dynamics, the study of a dynamical system of three gravitationally interacting point masses has fascinated researchers for centuries. In this study one of these masses is so much smaller (called infinitesimal mass $m_{3}, m_{3} \ll m_{1}, m_{2}$ ) than the other two masses $m_{1}$ and $m_{2}\left(m_{1}>m_{2}\right)$ (called primary and secondary body respectively) and has a negligible effect on their motion, known as restricted three-body problem (R3BP). This system, called the circular restricted three-body problem (CR3BP) or elliptic restricted three-body problem (ER3BP), depending on whether the primaries execute circular or elliptic orbit around their common centre of mass respectively.

The R3BP possesses five libration points: three such points are on the line joining the primaries called collinear points while, two (triangular points) form equilateral triangles with the primaries. The collinear points are however generally unstable, while the triangular points are stable for $0<\mu<\mu_{C}$ and unstable for $\mu_{C} \leq \mu \leq \frac{1}{2}$, where the critical mass parameter $\mu_{C}=0.03852 \ldots$ and mass ratio $\mu=\frac{m_{2}}{m_{1}+m_{2}} \leq \frac{1}{2}[1]$. The solution of this system has been developed over centuries from [1-5] and others.

The radiation pressure force is the second most powerful potential component after the gravitational influence. Radzievskii [6] formulated the photogravitational CR3BP in the cases of the Sun-planet-particle and Galaxy kernel-Sun-Particle. He found that an allowance for direct solar radiation pressure results in a change in the positions of the libration points. This has been intensively studied over five decades, by several researchers like [7-15], since a large percentage of astronomical bodies are emitters of radiation.

The classical R3BP considers the shape of the finite bodies as purely spherical, but it has been proved that astronomical bodies are not purely spherical [16, 17, 18, 19, 20, 21, 22, 23, 24]. They are either oblate spheroid or triaxial rigid bodies. For instance, the Sun, Jupiter, Earth, Neutron star, Black dwarfs e.t.c. are oblate in shape, while the moons of the Earth, Pluto and its moon Charon are triaxial rigid bodies. 
A vast number of researchers [25-32 and others] have included the effects of perturbing forces of oblateness, triaxiality and radiation pressure of the primaries in their studies.

The orbits of the celestial bodies are mostly elliptic not circular. Thus, the study of the ER3BP has significant effects. This has motivated $[1,7,33,8,34,11,12,35,13,36]$ to study ER3BP in different perspectives. [37, 15] and others, have investigated the collinear libration points of the circular and elliptical restricted three-body problem under different assumptions. [13, 36, 38] have shown that, the positions of the collinear libration points are affected by the potential due to eccentricity, radiation pressure, oblateness, and triaxiality of the primary bodies.

Our aim in this work is to investigate the effects of oblateness of the primary body and the luminosity and triaxiality of the secondary body on the locations as well as linear stability of the collinear libration points. This study is applied in the neighborhood of two binaries CEN X-4 and PSR 1903+0327 barrowed from Umar and Hussain [24].

The rest of this work is organized as follows: Section 2 presents the equations of motion; Sections 3 and 4 describes the positions and establishes the linear stability of the collinear libration points; Section 5 contains numerical application of the problem. Lastly, in section 6 we have discussed the results obtained.

\section{Equations of Motion}

The equations of motion of a test particle in the ER3BP with a bigger oblate primary and a triaxial as well as radiating secondary in dimensionless-pulsating coordinate $\operatorname{system}(\xi, \eta, \zeta)$ are given in [24] as:

$$
\begin{aligned}
& \xi^{\prime \prime}-2 \eta^{\prime}=\Omega_{\xi}^{*} \\
& \eta^{\prime \prime}+2 \xi^{\prime}=\Omega_{\eta}^{*} \\
& \zeta^{\prime \prime}=\Omega_{\zeta}^{*},
\end{aligned}
$$

where, the prime in equation (1) represents differentiation with respect to the eccentric anomaly $E$ ( $E$ is an angular parameter that defines the position of a body that is moving along an elliptic Keplerian orbit, see [11] for more details), $\Omega^{*}$ is the potential-like function defined by:

$$
\Omega^{*}=\left(1-e^{2}\right)^{-1 / 2}\left[\frac{\xi^{2}+\eta^{2}}{2}+\frac{1}{n^{2}}\left\{\frac{(1-\mu)}{r_{1}}+\frac{\mu q_{2}}{r_{2}}+\frac{(1-\mu) A}{2 r_{1}^{3}}+\frac{\mu q_{2}\left(2 \sigma_{1}-3 \sigma_{2}\right)}{2 r_{2}^{3}}-\frac{3 \mu q_{2}\left(\sigma_{1}-\sigma_{2}\right) \eta^{2}}{2 r_{2}^{5}}\right\}\right] .
$$

Where $r_{1}=\sqrt{(\xi+\mu)^{2}+\eta^{2}+\zeta^{2}}$ and $r_{2}=\sqrt{(\xi+\mu-1)^{2}+\eta^{2}+\zeta^{2}}$ are the distances of an infinitesimal from the primary and secondary bodies respectively. Also $a$ and $e$ are the semi-major axis and eccentricity of the orbit of the primaries respectively, $n$ is the perturbed mean motion of the primaries. While $q_{2}$ is the radiation pressure factor of the secondary body which is given by $F_{g}-F_{p}=F_{g}\left(1-\frac{F_{p}}{F_{g}}\right)=q_{2} F_{g}$, where $q_{2}=1-\frac{F_{p}}{F_{g}}$ such that $0<1-q_{2}=\delta \ll 1 \quad$ (Radzievskii [6]), where $F_{g}$ and $F_{p}$ are respectively the gravitational and radiation pressure forces. Additionally, $A=\frac{a_{1}^{2}-a_{3}^{2}}{5 R^{2}} \ll 1, \sigma_{1}=\frac{b_{1}^{2}-b_{3}^{2}}{5 R^{2}} \ll 1$ and $\sigma_{2}=\frac{b_{1}^{2}-b_{3}^{2}}{5 R^{2}} \ll 1$, where $a_{1}$ and $a_{3}$ are respectively the equatorial and polar radii of the primary body, $b_{1}, b_{2}$ and $b_{3}$ are the length of its semi-axes of the secondary body, and $R$ is the dimensional distance between the primaries. 


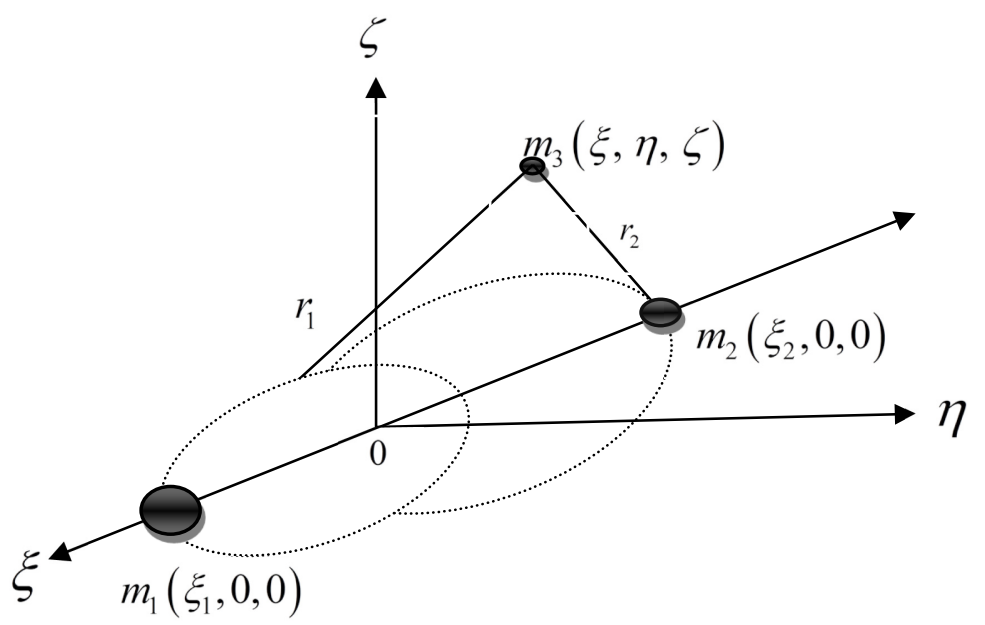

Figure 1. Primary $m_{1}$ and secondary $m_{2}$ moving in elliptic orbits about their common centre of mass (origin) unperturbed by the infinitesimal mass $m_{3}$ in the ER3BP

\subsection{Derivation of the perturbed mean motion}

In the case of elliptic orbit, the distance between the primaries is $r=\frac{a\left(1-e^{2}\right)}{1+e \cos v}$ and the mean distance between them is given by

$$
\frac{1}{2 \pi} \int_{0}^{2 \pi} r d v=\frac{a\left(1-e^{2}\right)}{\left(1+e^{2}\right)^{1 / 2}} .
$$

The orbit of $m_{1}$ and $m_{2}$ with respect to the centre of mass, with semi-major axes $a_{1}=a m_{2}$ and $a_{2}=a m_{1}$ respectively, have the same eccentricity with [1].

Their equations are given by

$$
\frac{n^{2} a m_{1}\left(1-e^{2}\right)}{\left(1+e^{2}\right)^{1 / 2}}=k^{2} m_{2}\left(1+\frac{3 A}{2}+\frac{3\left(2 \sigma_{1}-\sigma_{2}\right)}{2}\right),
$$

and

$$
\frac{n^{2} a m_{2}\left(1-e^{2}\right)}{\left(1+e^{2}\right)^{1 / 2}}=k^{2} m_{1}\left(1+\frac{3 A}{2}+\frac{3\left(2 \sigma_{1}-\sigma_{2}\right)}{2}\right) .
$$

Adding equations (3) together we have

$$
n^{2}=\frac{k^{2}\left(1+e^{2}\right)^{1 / 2}}{a\left(1-e^{2}\right)}\left(1+\frac{3 A}{2}+\frac{3\left(2 \sigma_{1}-\sigma_{2}\right)}{2}\right) .
$$

Here, we adopt that, the sum of the masses of the primaries and the distances between them as the units of mass and length. We choose the unit of time such as to make $k^{2}=1$, where $k$ is the Gaussian constant of gravitation. Hence,

$$
n^{2}=\frac{k^{2}\left(1+e^{2}\right)^{1 / 2}}{a\left(1-e^{2}\right)}\left(1+\frac{3 A}{2}+\frac{3\left(2 \sigma_{1}-\sigma_{2}\right)}{2}\right) .
$$

Making the simplification of the above $n^{2}$, by rejecting the second and higher order terms of $e^{2}$, we get

$$
n^{2}=\frac{1}{a}\left(1+\frac{3 e^{2}}{2}+\frac{3 A}{2}+\frac{3\left(2 \sigma_{1}-\sigma_{2}\right)}{2}\right) .
$$




\section{Positions of the Collinear Libration Points}

To obtain the collinear libration points, we obtain the first partial derivatives of equation (2) with respect to $\xi, \eta$ and $\zeta$ respectively, and equate them to zero. That is, $\Omega_{\xi}^{*}=\Omega_{\eta}^{*}=\Omega_{\zeta}^{*}=0$. Since the collinear libration points lies on the $\xi$-axis, it implies that $\zeta=\eta=0$. i.e.

$$
\begin{aligned}
\Omega_{\xi}^{*} & =\left(1-e^{2}\right)^{-1 / 2}\left[\xi-\frac{1}{n^{2}}\left\{\frac{(1-\mu)(\xi+\mu)}{r_{1}^{3}}+\frac{3(1-\mu)(\xi+\mu) A}{2 r_{1}^{5}}+\frac{\mu q_{2}(\xi+\mu-1)}{r_{2}^{3}}\right.\right. \\
& \left.\left.+\frac{3 \mu q_{2}(\xi+\mu-1)\left(2 \sigma_{1}-\sigma_{2}\right)}{2 r_{2}^{5}}+\frac{15 \mu q_{2}\left(\sigma_{1}-\sigma_{2}\right)(\xi+\mu-1) \eta^{2}}{2 r_{2}^{7}}\right\}\right]=0, \\
& \Omega_{\eta}^{*}=\left(1-e^{2}\right)^{-1 / 2} \eta\left[1-\frac{1}{n^{2}}\left\{\frac{(1-\mu)}{r_{1}^{3}}+\frac{3(1-\mu) A}{2 r_{1}^{5}}+\frac{\mu q_{2}}{r_{2}^{3}}+\frac{3 \mu q_{2}\left(4 \sigma_{1}-3 \sigma_{2}\right)}{2 r_{2}^{5}}-\frac{15 \mu q_{2}\left(\sigma_{1}-\sigma_{2}\right) \eta^{2}}{2 r_{2}^{7}}\right\}\right]=0, \\
& \Omega_{\zeta}^{*}=\left(1-e^{2}\right)^{-1 / 2}\left[-\frac{\zeta}{n^{2}}\left\{\frac{(1-\mu)}{r_{1}^{3}}+\frac{3(1-\mu) A}{2 r_{1}^{5}}+\frac{\mu q_{2}}{r_{2}^{3}}+\frac{3 \mu q_{2}\left(2 \sigma_{1}-\sigma_{2}\right)}{2 r_{2}^{5}}-\frac{15 \mu q_{2}\left(\sigma_{1}-\sigma_{2}\right) \eta^{2}}{2 r_{2}^{7}}\right\}\right]=0 .
\end{aligned}
$$

Since, $\eta=\zeta=0$, using equation (3), the first equation of (5), reduces to

$$
\xi n^{2}-\frac{(1-\mu)(\xi+\mu)}{|\xi+\mu|^{3}}-\frac{3(1-\mu)(\xi+\mu) A}{2|\xi+\mu|^{5}}-\frac{\mu q_{2}(\xi+\mu-1)}{|\xi+\mu-1|^{3}}-\frac{3 \mu q_{2}(\mu+\mu-1)\left(2 \sigma_{1}-\sigma_{2}\right)}{2|\xi+\mu-1|^{5}}=0,
$$

and equation (3) becomes

$$
r_{1}^{2}=(\xi+\mu)^{2} ; r_{2}^{2}=(\xi+\mu-1)^{2}
$$

Solving equation (6) using equation (7) with $\xi_{1}=-\mu$ and $\xi_{2}=1-\mu$ we obtain

$$
\begin{aligned}
& 2 \xi n^{2}\left(\xi-\xi_{1}\right)^{4}\left(\xi-\xi_{2}\right)^{4}-2(1-\mu)\left(\xi-\xi_{1}\right)^{2}\left(\xi-\xi_{2}\right)^{4}-3(1-\mu) A\left(\xi-\xi_{2}\right)^{4}-2 \mu q_{2}\left(\xi-\xi_{1}\right)^{4}\left(\xi-\xi_{2}\right)^{2} \\
& -3 \mu q_{2}\left(2 \sigma_{1}-\sigma_{2}\right)\left(\xi-\xi_{1}\right)^{4}=0 .
\end{aligned}
$$

The orbital plane on $\xi$-axis is divided into three parts; $\xi>\xi_{2}, \xi_{1}<\xi<\xi_{2}$, and $\xi_{1}>\xi$ with respect to the primaries, which corresponds to the collinear points $L_{i}(i=1,2,3)$.

\subsection{Position of $L_{1}\left(\xi>\xi_{2}\right)$}

Let the collinear libration point $L_{1}$ be on the right hand side of the smaller primary at a distance $\rho$ from it on the $\xi$-axis.

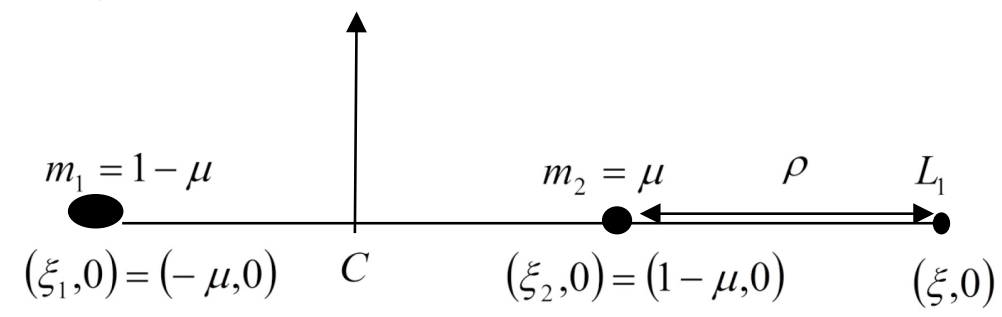

Figure 2. Position of collinear libration point $L_{1}$

In the interval $\left(\xi>\xi_{2}\right)$, we let $\xi-\xi_{2}=\rho, \xi-\xi_{1}=1+\rho \Rightarrow \xi=1+\rho+\xi_{1}$. Since the distance between the primaries is unity, using equation (7) we have

$$
r_{1}=1+\rho \text { and } r_{2}=\rho .
$$


Substituting equation (9) in the equation (8) we have

$$
\begin{aligned}
& 2 n^{2}(1+\rho-\mu)(1+\rho)^{4} \rho^{4}-2(1-\mu)(1+\rho)^{2} \rho^{4}-2 \mu q_{2}(1+\rho)^{4} \rho^{2}-3(1-\mu) A \rho^{4} \\
& -3 \mu q_{2}\left(2 \sigma_{1}-\sigma_{2}\right)(1+\rho)^{4}=0 .
\end{aligned}
$$

Hence, expanding equation (10) we get

$$
\begin{aligned}
& 2 n^{2} \rho^{9}+2 n^{2}(5-\mu) \rho^{8}+2 n^{2}(2(5-2 \mu)) \rho^{7}+2\left(2 n^{2}(5-3 \mu)-\left(1-\mu+\mu q_{2}\right)\right) \rho^{6} \\
& +\left(2 n^{2}(5-4 \mu)-4\left(1-\mu+2 \mu q_{2}\right)\right) \rho^{5}+\left(2 n^{2}(1-\mu)-2\left(1-\mu+\mu q_{2}\right)-3(1-\mu) A-6 \mu q_{2} \sigma_{1}+3 \mu q_{2} \sigma_{2}\right) \rho^{4} \\
& +\left(-4\left(2 \mu q_{2}+6 \mu q_{2} \sigma_{1}-3 \mu q_{2} \sigma_{2}\right)\right) \rho^{3}+\left(-2\left(\mu q_{2}+18 \mu q_{2} \sigma_{1}-9 \mu q_{2} \sigma_{2}\right)\right) \rho^{2} \\
& +\left(-12\left(2 \mu q_{2} \sigma_{1}-\mu q_{2} \sigma_{2}\right)\right) \rho-\left(3\left(2 \mu q_{2} \sigma_{1}-\mu q_{2} \sigma_{2}\right)\right)=0 .
\end{aligned}
$$

3.2 Position of $L_{2}\left(\xi_{1}<\xi<\xi_{2}\right)$

Let the collinear libration point $L_{2}$ be on the left hand side of the smaller primary at a distance $\rho$ from it on the $\xi$-axis.

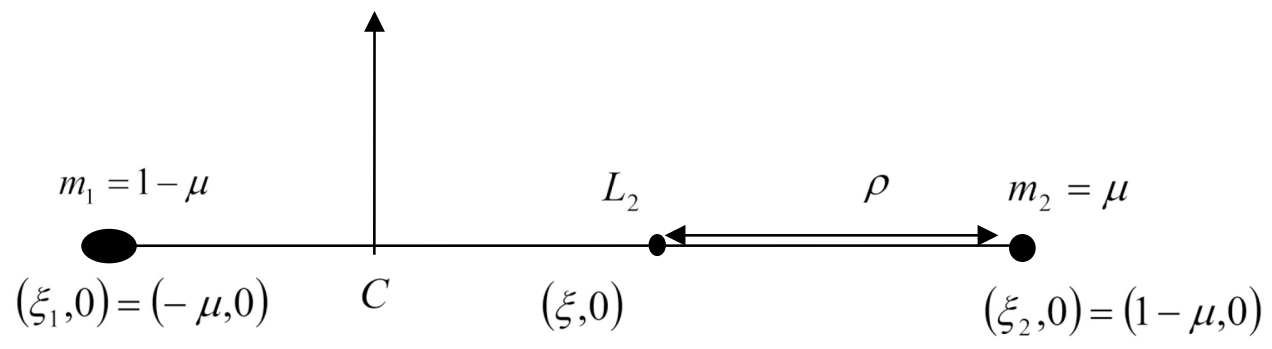

Figure 3. Position of collinear libration point $L_{2}$

In the interval $L_{2}\left(\xi_{1}<\xi<\xi_{2}\right)$, we let

$$
\xi_{2}-\xi=\rho ; \xi-\xi_{1}=1-\rho \Rightarrow \xi=1-\rho-\mu ; \text { and } r_{1}=1-\rho, r_{2}=\rho .
$$

Using equation (12) in the equation (8) we have

$$
\begin{aligned}
& 2 n^{2}(1-\rho-\mu)(1-\rho)^{4} \rho^{4}-2(1-\mu)(1-\rho)^{2} \rho^{4}-2 \mu q_{2}(1-\rho)^{4} \rho^{2}-3(1-\mu) A \rho^{4} \\
& +3 \mu q_{2}\left(2 \sigma_{1}-\sigma_{2}\right)(1-\rho)^{4}=0 .
\end{aligned}
$$

Hence, expanding equation (13) we get

$$
\begin{aligned}
& -2 n^{2} \rho^{9}+2 n^{2}(5-\mu) \rho^{8}+2 n^{2}(-2(5-2 \mu)) \rho^{7}+2\left(2 n^{2}(5-3 \mu)-\left(1+\mu-\mu q_{2}\right)\right) \rho^{6} \\
& +\left(-2 n^{2}(5-4 \mu)+4\left(1+\mu-2 \mu q_{2}\right)\right) \rho^{5}+\left(2 n^{2}(1-\mu)-2\left(1-\mu-6 \mu q_{2}\right)-3(1-\mu) A+6 \mu q_{2} \sigma_{1}-3 \mu q_{2} \sigma_{2}\right) \rho^{4} \\
& +\left(-4\left(2 \mu q_{2}+6 \mu q_{2} \sigma_{1}-3 \mu q_{2} \sigma_{2}\right)\right) \rho^{3}+\left(2\left(\mu q_{2}+18 \mu q_{2} \sigma_{1}-9 \mu q_{2} \sigma_{2}\right)\right) \rho^{2} \\
& +\left(-12\left(2 \mu q_{2} \sigma_{1}-\mu q_{2} \sigma_{2}\right)\right) \rho+\left(-3\left(-2 \mu q_{2} \sigma_{1}+\mu q_{2} \sigma_{2}\right)\right)=0 .
\end{aligned}
$$

\subsection{Position of $L_{3}\left(\xi_{1}>\xi\right)$}

Let the collinear libration point $L_{3}$ be on the left hand side of the bigger primary at a distance $1-\rho$ from it on the $\xi$-axis. 


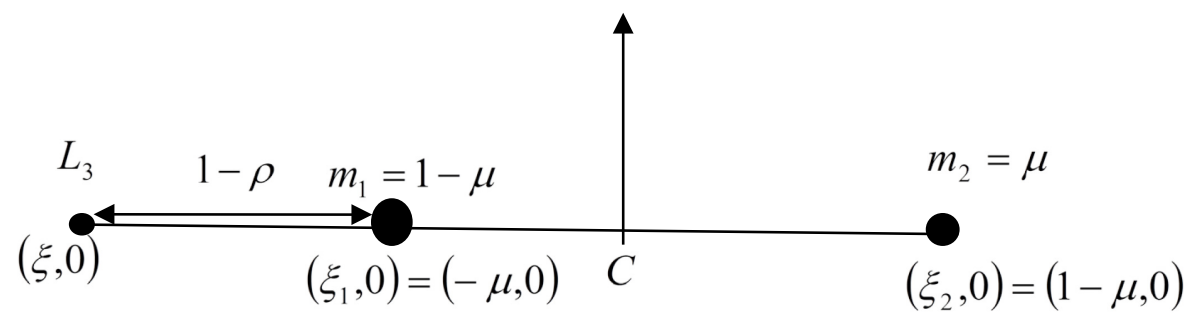

Figure 4. Position of collinear libration point $L_{3}$

Lastly, in the interval $L_{3}\left(\xi_{1}>\xi\right)$, we let

$$
\xi_{1}-\xi=1-\rho ; \xi_{2}-\xi=2-\rho, \text { and } r_{1}=1-\rho ; r_{2}=2-\rho \text {. }
$$

Using equation (15) in the equation (8) we have

$$
\begin{aligned}
& 2 n^{2}(\rho-\mu-1)(1-\rho)^{4}(2-\rho)^{4}+2(1-\mu)(1-\rho)^{2}(2-\rho)^{4}+2 \mu q_{2}(2-\rho)^{2}(1-\rho)^{4}+3(1-\mu) A(2-\rho)^{4} \\
& +3 \mu q_{2}\left(2 \sigma_{1}-\sigma_{2}\right)(1-\rho)^{4}=0 .
\end{aligned}
$$

Hence, expanding equation (16) we get

$$
\begin{aligned}
& 2 n^{2} \rho^{9}+2 n^{2}(-13-\mu) \rho^{8}+4 n^{2}(2(37+6 \mu)) \rho^{7}+2\left(-2 n^{2}(121-31 \mu)+\left(1-\mu+\mu q_{2}\right)\right) \rho^{6} \\
& +2\left(n^{2}(501+180 \mu)-2\left(5-5 \mu+4 \mu q_{2}\right)\right) \rho^{5}+\left(-2 n^{2}(681-321 \mu)+2\left(41-41 \mu+26 \mu q_{2}\right)+3(1-\mu) A\right. \\
& \left.+6 \mu q_{2} \sigma_{1}-3 \mu q_{2} \sigma_{2}\right) \rho^{4}+\left(2 n^{2}(609+360 \mu)-88\left(2-2 \mu+\mu q_{2}\right)-24(1-\mu) A-12\left(2 \mu q_{2} \sigma_{1}-\mu q_{2} \sigma_{2}\right)\right) \rho^{3} \\
& +\left(-2 n^{2}(345+249 \mu)+2\left(104-104 \mu+41 \mu a_{2}\right)-72(1-\mu) A+18\left(2 \mu q_{2} \sigma_{1}-\mu q_{2} \sigma_{2}\right)\right) \rho^{2} \\
& +\left(2 n^{2}(112+96 \mu)-2\left(64-64 \mu+20 \mu q_{2}\right)-96(1-\mu) A-12\left(2 \mu q_{2} \sigma_{1}-\mu q_{2} \sigma_{2}\right)\right) \rho \\
& +2\left(-2 n^{2}(8(1+\mu))+4\left(4-4 \mu+\mu q_{2}\right)+24(1-\mu) A\right)+3\left(2 \mu q_{2} \sigma_{1}-\mu q_{2} \sigma_{2}\right)=0 .
\end{aligned}
$$

Equations (11), (14) and (17) are ninth degree equations and some of these equations ((11), (14) and (17)) have more than one positive real root, but in each case there is only one physically acceptable root which corresponds to one of the three collinear libration points $L_{i}(i=1,2,3)$. This is confirmed by Yamada and Asada [30].

\section{Stability of the Collinear Libration Points}

To study the stability of the collinear libration points $L_{i}(i=1,2,3)$, we consider the characteristic equation of the system in Singh and Umar [11] given by:

$$
\lambda^{4}-\left(\Omega_{\xi \xi}^{* 0}+\Omega_{\eta \eta}^{* 0}-4\right) \lambda^{2}+\Omega_{\xi \xi}^{* 0} \Omega_{\eta \eta}^{* 0}-\left(\Omega_{\xi \eta}^{* 0}\right)^{2}=0 .
$$

Here, we obtain the points corresponding to the collinear by taking the second partial derivatives of equation (2), with $\eta=0$. Thus we have

$$
\begin{aligned}
& \Omega_{\xi \xi}^{* 0}=\left(1-e^{2}\right)^{-1 / 2}\left[1+\frac{2}{n^{2}}\left\{\frac{(1-\mu)}{|\xi+\mu|^{3}}+\frac{\mu q_{2}}{|\xi+\mu-1|^{3}}+\frac{3(1-\mu) A}{|\xi+\mu|^{5}}+\frac{3 \mu q_{2}\left(2 \sigma_{1}-\sigma_{2}\right)}{|\xi+\mu-1|^{5}}\right\},\right. \\
& \Omega_{\eta \eta}^{* 0}=\left(1-e^{2}\right)^{-1 / 2}\left[1-\frac{1}{n^{2}}\left\{\frac{(1-\mu)}{|\xi+\mu|^{3}}+\frac{3(1-\mu) A}{|\xi+\mu-1|^{3}}+\frac{\mu q_{2}}{2|\xi+\mu|^{5}}+\frac{3 \mu q_{2}\left(4 \sigma_{1}-3 \sigma_{2}\right)}{2|\xi+\mu-1|^{5}}\right\},\right. \\
& \Omega_{\zeta \zeta}^{* 0}=0 .
\end{aligned}
$$


Since $\Omega_{\xi \xi}^{* 0}$ contains only positive terms, implies that

$$
\Omega_{\xi \xi}^{* 0}>0
$$

\subsection{Stability of $L_{1}\left(\xi>\xi_{2}\right)$}

In the first interval, we have

$$
r_{1}=(\xi+\mu) \Rightarrow \xi=\left(r_{1}-\mu\right) \text { and } r_{2}=(\xi+\mu-1) .
$$

Substituting equation (21) in the equation (6), we obtain

$$
\frac{(1-\mu)}{r_{1}^{2}}=\xi n^{2}-\frac{\mu q_{2}}{r_{2}^{2}}-\frac{3(1-\mu) A}{2 r_{1}^{4}}-\frac{3 \mu q_{2}\left(2 \sigma_{1}-\sigma_{2}\right)}{2 r_{2}^{4}} .
$$

Substituting equation (22) in the second equation of (19), we have

$$
\begin{aligned}
\Omega_{\eta \eta}^{* 0}= & \left(1-e^{2}\right)^{-1 / 2}\left[1-\frac{1}{n^{2}}\left\{\frac{1}{r_{1}}\left(\xi n^{2}-\frac{\mu q_{2}}{r_{2}^{2}}-\frac{3(1-\mu) A}{2_{1}^{4}}-\frac{3 \mu q_{2}\left(2 \sigma_{1}-\sigma_{2}\right)}{2 r_{2}^{4}}\right)\right.\right. \\
& \left.+\frac{3(1-\mu) A}{r^{3}}+\frac{\mu q_{2}}{2 r^{5}}+\frac{3 \mu q_{2}\left(4 \sigma_{1}-3 \sigma_{2}\right)}{2 r^{5}}\right\} .
\end{aligned}
$$

Implies that

$$
\Omega_{\eta \eta}^{* 0}=\left(1-e^{2}\right)^{-1 / 2}\left[\frac{\mu}{r_{1}}+\frac{1}{n^{2}}\left\{\frac{\mu q_{2}}{r_{2}^{2}}\left(\frac{1}{r_{1}}-\frac{1}{r_{2}}\right)+\frac{3 \mu q_{2}}{2 r_{2}^{4}}\left(\frac{\left(2 \sigma_{1}-\sigma_{2}\right)}{r_{1}}-\frac{\left(4 \sigma_{1}-3 \sigma_{2}\right)}{r_{2}}\right)\right\} .\right.
$$

Thus, $\Omega_{\eta \eta}^{* 0}<0$, since $\mu<\frac{1}{2}, A, \sigma_{i}(i=1,2)<<1, r_{1}>1$ and $r_{2}<1$.

4.2 Stability of $L_{2}\left(\xi_{1}<\xi<\xi_{2}\right)$

In the second interval, we have

$$
r_{1}=(\xi+\mu) \Rightarrow \xi=\left(r_{1}-\mu\right) \text { and } r_{2}=-(\xi+\mu-1) .
$$

Substituting equation (25) in the equation (6), we obtain

$$
\frac{(1-\mu)}{r_{1}^{2}}=\xi n^{2}+\frac{\mu q_{2}}{r_{2}^{2}}-\frac{3(1-\mu) A}{2 r_{1}^{4}}+\frac{3 \mu q_{2}\left(2 \sigma_{1}-\sigma_{2}\right)}{2 r_{2}^{4}} .
$$

Substituting equation (26) in the second equation of (19), we have

$$
\Omega_{\eta \eta}^{* 0}=\left(1-e^{2}\right)^{-1 / 2}\left[-\frac{\mu}{r_{1}}+\frac{1}{n^{2}}\left\{\frac{\mu q_{2}}{r_{2}^{2}}\left(\frac{1}{r_{1}}-\frac{1}{r_{2}}\right)+\frac{3 \mu q_{2}}{2 r_{2}^{4}}\left(\frac{\left(2 \sigma_{1}-\sigma_{2}\right)}{r_{1}}+\frac{\left(4 \sigma_{1}-3 \sigma_{2}\right)}{r_{2}}\right)\right\}\right] \text {. }
$$

Thus, $\Omega_{\eta \eta}^{* 0}<0$, since $\mu<\frac{1}{2}, A, \sigma_{i}(i=1,2)<<1, r_{1}>1$ and $r_{2}<1$.

\subsection{Stability of $L_{3}\left(\xi_{1}>\xi\right)$}

In the last interval, we have

$$
r_{1}=-(\xi+\mu) \Rightarrow \xi=-\left(r_{1}+\mu\right) \text { and } r_{2}=-(\xi+\mu-1) .
$$

Substituting equation (28) in the equation (6), we obtain 


$$
\frac{(1-\mu)}{r_{1}^{2}}=-\xi n^{2}-\frac{\mu q_{2}}{r_{2}^{2}}-\frac{3(1-\mu) A}{2 r_{1}^{4}}-\frac{3 \mu q_{2}\left(2 \sigma_{1}-\sigma_{2}\right)}{2 r_{2}^{4}} .
$$

Substituting equation (29) in the second equation of (19), we have

$$
\Omega_{\eta \eta}^{* 0}=\left(1-e^{2}\right)^{-1 / 2}\left[-\frac{\mu}{r_{1}}+\frac{1}{n^{2}}\left\{\frac{\mu q_{2}}{r_{2}^{2}}\left(\frac{1}{r_{1}}-\frac{1}{r_{2}}\right)+\frac{3 \mu q_{2}}{2 r_{2}^{4}}\left(\frac{\left(2 \sigma_{1}-\sigma_{2}\right)}{r_{1}}-\frac{\left(4 \sigma_{1}-3 \sigma_{2}\right)}{r_{2}}\right)\right\}\right] .
$$

Thus, $\Omega_{\eta \eta}^{* 0}<0$, since $\mu<\frac{1}{2}, A, \sigma_{i}(i=1,2)<<1, r_{1}>1$ and $r_{2}<1$.

Clearly, $\Omega_{\xi \xi}^{* 0}>0, \Omega_{\eta \eta}^{* 0}<0$, and $\Omega_{\xi \eta}^{* 0}=0$.

Since, $\Omega_{\xi \xi}^{* 0} \Omega_{\eta \eta}^{* 0}-\left(\Omega_{\xi \eta}^{* 0}\right)^{2}<0$ the discriminant of equation (18) is positive and its characteristic roots can be expressed as:

$$
\lambda_{1,2}= \pm h, \lambda_{3,4}= \pm i h^{\prime}
$$

where $h$ and $h^{\prime}$ are real.

Hence, we conclude that, the collinear libration points are unstable (due to the nature of the characteristic roots of equations 31), despite the fact that perturbations on account of oblateness, eccentricity, triaxiality and luminosity were introduced.

\section{Numerical Application}

We obtain numerically, the roots and positions of the collinear libration points $L_{i}(i=1,2,3)$ of the low mass X-ray binary (LMXB) CEN X-4 and $L_{1}$ of the high mass X-ray binary (HMXB) PSR J1903+0327. The radiation pressure factor $q_{2}$ of the smaller primary is computed using $q_{2}=1-(\Phi \tau L / \vartheta \rho m)$ on the basis of Stefan-Boltzmann's law with $\tau=1$; where $m$ and $L$ are the mass and luminosity respectively; $\vartheta$ and $\rho$ are the radius and density of a moving test particle; $\tau$ is the radiation pressure efficiency of a star; $\Phi=(3 / 16 \pi C G)$. In the C.G.S. system $\Phi=2.9838 \times 10^{-5}$ Table 1 contains the numerical data of the binaries, while the effects of triaxiality, oblateness, eccentricity and semi-major axis are given in Tables 2-17. $\rho L_{i}(i=1,2,3)$ are the roots of the equation (11), (14) and (17) which corresponds to the position of the collinear libration point $L_{i}(i=1,2,3)$. These effects are shown graphically in Figs. 5-10.

Table 1. Relevant Numerical data

\begin{tabular}{|l|cc|l|c|c|}
\hline Binary System & \multicolumn{2}{|c|}{ Masses $\left(\mathrm{M}_{\mathrm{SUN}}\right)$} & Luminosity & Mass ratio & Radiation pressure \\
& $m_{1}$ & $m_{2}$ & $\left(L_{\text {sun }}\right)$ & $(\mu)$ & $\left(q_{2}\right)$ \\
\hline CEN-X4 & 1.9996 & 0.0801 & 0.2466 & 0.038515 & 0.999300 \\
\hline PSR 1903+0327 & 1.6700 & 1.1000 & 2.5000 & 0.397112 & 0.997581 \\
\hline
\end{tabular}

Table 2. Effect of triaxiality on $L_{1}$ of CEN X-4 for $A=0.01, e=0.2 \& a=0.8$

\begin{tabular}{|l|l|l|l|l|l|l|l|l|}
\hline$\sigma_{1}$ & $\sigma_{2}$ & $\rho L_{1}$ & $L_{1}$ & $\Omega_{\xi \xi}^{* 0}$ & $\Omega_{\eta \eta}^{* 0}$ & $\Omega_{\xi \xi}^{* 0} \Omega_{\eta \eta}^{* 0}$ & $\lambda_{1,2}$ & $\lambda_{3,4}$ \\
\hline 0.01 & 0.0075 & 0.204998 & 1.166483 & 11.7813 & -3.6435 & -42.9246 & \pm 2.9900 & $\pm 2.1913 i$ \\
\hline 0.02 & 0.0150 & 0.220476 & 1.181961 & 12.3704 & -3.6767 & -45.4819 & \pm 3.0802 & $\pm 2.1895 i$ \\
\hline 0.03 & 0.0225 & 0.231659 & 1.193144 & 12.6887 & -3.6879 & -46.7945 & \pm 3.1279 & $\pm 2.1870 i$ \\
\hline 0.04 & 0.0333 & 0.238318 & 1.199803 & 13.3604 & -3.8456 & -51.3791 & \pm 3.2307 & $\pm 2.2187 i$ \\
\hline
\end{tabular}


Table 3. Effect of oblateness on $L_{1}$ of CEN X-4 for $\sigma_{1}=0.01, \sigma_{1}=0.0075, e=0.2 \& a=0.8$

\begin{tabular}{|l|l|l|l|l|l|l|l|}
\hline$A$ & $\rho L_{1}$ & $L_{1}$ & $\Omega_{\xi \xi}^{* 0}$ & $\Omega_{\eta \eta}^{* 0}$ & $\Omega_{\xi \xi}^{* 0} \Omega_{\eta \eta}^{* 0}$ & $\lambda_{1,2}$ & $\lambda_{3,4}$ \\
\hline 0.0001 & 0.206037 & 1.167522 & 11.7123 & -3.6205 & -42.4049 & \pm 2.9785 & $\pm 2.1863 i$ \\
\hline 0.001 & 0.205942 & 1.167427 & 11.7186 & -3.6226 & -42.4517 & \pm 2.9710 & $\pm 2.1867 i$ \\
\hline 0.01 & 0.204998 & 1.166483 & 11.7813 & -3.6435 & -42.9246 & \pm 2.9899 & $\pm 2.1913 i$ \\
\hline 0.1 & 0.196481 & 1.157966 & 12.3702 & -3.8383 & -47.4799 & \pm 3.0854 & $\pm 2.2333 i$ \\
\hline
\end{tabular}

Table 4. Effect of eccentricity on $L_{1}$ of CEN X-4 for $\sigma_{1}=0.01, \sigma_{2}=0.0075, a=0.8 \& A=0.1$

\begin{tabular}{|r|l|l|l|l|l|l|l|}
\hline \multicolumn{1}{|l|}{$e$} & $\rho L_{1}$ & $L_{1}$ & $\Omega_{\xi \xi}^{* 0}$ & $\Omega_{\eta \eta}^{* 0}$ & $\Omega_{\xi \xi}^{* 0} \Omega_{\eta \eta}^{* 0}$ & $\lambda_{1,2}$ & $\lambda_{3,4}$ \\
\hline 0.99 & 0.141468 & 1.102953 & 30.0000 & -10.1265 & -306.835 & \pm 5.2326 & $\pm 3.3476 i$ \\
\hline 0.9 & 0.147169 & 1.108654 & 26.8772 & -8.9379 & -240.224 & \pm 4.8953 & $\pm 3.1661 i$ \\
\hline 0.8 & 0.153982 & 1.115467 & 23.5288 & -7.7715 & -182.853 & \pm 4.5413 & $\pm 2.9776 i$ \\
\hline 0.7 & 0.161255 & 1.122740 & 20.6373 & -6.7605 & -139.518 & \pm 4.2120 & $\pm 2.8043 i$ \\
\hline 0.6 & 0.168872 & 1.130357 & 18.1826 & -5.8990 & -107.253 & \pm 3.9110 & $\pm 2.6480 i$ \\
\hline 0.5 & 0.176617 & 1.138102 & 16.1453 & -5.1802 & -83.6366 & \pm 3.6426 & $\pm 2.5107 i$ \\
\hline 0.4 & 0.184143 & 1.145628 & 14.5078 & -4.6001 & -66.7372 & \pm 3.4119 & $\pm 2.3944 i$ \\
\hline 0.3 & 0.190962 & 1.152447 & 13.2541 & -4.1540 & -55.0570 & \pm 3.2243 & $\pm 2.3013 i$ \\
\hline
\end{tabular}

Table 5. Effect of semi-major axis on $L_{1}$ of CEN X-4 for

$$
\sigma_{1}=0.03, \sigma_{2}=0.0225, e=0.2 \& A=0.01
$$

\begin{tabular}{|l|l|l|l|l|l|l|l|}
\hline$a$ & $\rho L_{1}$ & $L_{1}$ & $\Omega_{\xi \xi}^{* 0}$ & $\Omega_{\eta \eta}^{* 0}$ & $\Omega_{\xi \xi}^{* 0} \Omega_{\eta \eta}^{* 0}$ & $\lambda_{1,2}$ & $\lambda_{3,4}$ \\
\hline 0.95 & 0.2694570 & 1.230942 & 9.96159 & -2.7031 & -26.9273 & \pm 2.6586 & $\pm 1.9518 i$ \\
\hline 0.85 & 0.2438380 & 1.205323 & 11.6870 & -3.3287 & -38.9021 & \pm 2.9641 & $\pm 2.1042 i$ \\
\hline 0.75 & 0.2198450 & 1.181330 & 13.7948 & -4.0818 & -56.3083 & \pm 3.2993 & $\pm 2.2744 i$ \\
\hline 0.65 & 0.1917250 & 1.153210 & 18.3574 & -5.5677 & -103.860 & \pm 3.9282 & $\pm 2.5944 i$ \\
\hline 0.55 & 0.1752640 & 1.136749 & 19.5327 & -6.0922 & -118.996 & \pm 4.0751 & $\pm 2.6769 i$ \\
\hline 0.45 & 0.1537710 & 1.115256 & 23.5101 & -7.4651 & -175.506 & \pm 4.5360 & $\pm 2.9206 i$ \\
\hline 0.35 & 0.1320040 & 1.093489 & 28.7306 & -9.2548 & -265.895 & \pm 5.0781 & $\pm 3.2111 i$ \\
\hline
\end{tabular}

Table 6. Effect of triaxiality on $L_{2}$ of CEN X-4 for $A=0.01, e=0.2 \& a=0.8$

\begin{tabular}{|l|l|l|l|l|l|l|l|l|}
\hline$\sigma_{1}$ & $\sigma_{2}$ & $\rho L_{2}$ & $L_{2}$ & $\Omega_{\xi \xi}^{* 0}$ & $\Omega_{\eta \eta}^{* 0}$ & $\Omega_{\xi \xi}^{* 0} \Omega_{\eta \eta}^{* 0}$ & $\lambda_{1,2}$ & $\lambda_{3,4}$ \\
\hline 0.01 & 0.0075 & 0.269258 & 0.692227 & 7.6974 & -2.0948 & -16.1243 & \pm 2.2127 & $\pm 1.8148 i$ \\
\hline 0.02 & 0.0150 & 0.280140 & 0.681345 & 8.2380 & -2.2507 & -18.5411 & \pm 2.3265 & $\pm 1.8508 i$ \\
\hline 0.03 & 0.0225 & 0.289096 & 0.672389 & 8.6051 & -2.3555 & -20.2692 & \pm 2.4011 & $\pm 1.8750 i$ \\
\hline 0.04 & 0.0333 & 0.294860 & 0.666625 & 8.9664 & -2.4896 & -22.3223 & \pm 2.4744 & $\pm 1.9094 i$ \\
\hline
\end{tabular}

Table 7. Effect of oblateness on $L_{2}$ of CEN X-4 for $\sigma_{1}=0.01, \sigma_{1}=0.0075, e=0.2 \& a=0.8$

\begin{tabular}{|l|l|l|l|l|l|l|l|}
\hline$A$ & $\rho L_{2}$ & $L_{2}$ & $\Omega_{\xi \xi}^{* 0}$ & $\Omega_{\eta \eta}^{* 0}$ & $\Omega_{\xi \xi}^{* 0} \Omega_{\eta \eta}^{* 0}$ & $\lambda_{1,2}$ & $\lambda_{3,4}$ \\
\hline 0.0001 & 0.271975 & 0.689510 & 7.5258 & -2.0564 & -15.4761 & \pm 2.1764 & $\pm 1.8076 i$ \\
\hline 0.001 & 0.271721 & 0.689764 & 7.5418 & -2.0600 & -15.5360 & \pm 2.1798 & $\pm 1.8082 i$ \\
\hline 0.01 & 0.269258 & 0.692227 & 7.6974 & -2.0948 & -16.1243 & \pm 2.2127 & $\pm 1.8148 i$ \\
\hline 0.1 & 0.250085 & 0.711400 & 8.9170 & -2.3703 & -21.1360 & \pm 2.4584 & $\pm 1.8701 i$ \\
\hline
\end{tabular}


Table 8. Effect of eccentricity on $L_{2}$ of CEN X-4 for $\sigma_{1}=0.01, \sigma_{2}=0.0075, a=0.8 \& A=0.1$

\begin{tabular}{|r|l|l|l|l|l|l|l|}
\hline \multicolumn{1}{|l|}{$e$} & $\rho L_{2}$ & $L_{2}$ & $\Omega_{\xi \xi}^{* 0}$ & $\Omega_{\eta \eta}^{* 0}$ & $\Omega_{\xi \xi}^{* 0} \Omega_{\eta \eta}^{* 0}$ & $\lambda_{1,2}$ & $\lambda_{3,4}$ \\
\hline 0.99 & 0.339008 & 0.622477 & 6.4955 & -0.5647 & -3.6678 & \pm 1.7636 & $\pm 1.0860 i$ \\
\hline 0.9 & 0.324693 & 0.636792 & 6.4587 & -0.6900 & -4.4560 & \pm 1.7816 & $\pm 1.1849 i$ \\
\hline 0.8 & 0.309383 & 0.652102 & 6.5298 & -0.8599 & -5.6153 & \pm 1.8296 & $\pm 1.2952 i$ \\
\hline 0.7 & 0.295111 & 0.666374 & 6.7238 & -1.0666 & -7.1713 & \pm 1.9057 & $\pm 1.4052 i$ \\
\hline 0.6 & 0.282284 & 0.679201 & 7.0418 & -1.3094 & -9.2208 & \pm 2.0060 & $\pm 1.5138 i$ \\
\hline 0.5 & 0.271221 & 0.690264 & 7.4677 & -1.5815 & -11.8103 & \pm 2.1229 & $\pm 1.6188 i$ \\
\hline 0.4 & 0.262123 & 0.699362 & 7.9641 & -1.8669 & -14.8680 & \pm 2.2460 & $\pm 1.7168 i$ \\
\hline 0.3 & 0.255076 & 0.706409 & 8.4721 & -2.1403 & -18.1332 & \pm 2.3624 & $\pm 1.8025 i$ \\
\hline
\end{tabular}

Table 9. Effect of semi-major axis on $L_{2}$ of CEN X-4 for $\sigma_{1}=0.03, \sigma_{2}=0.0225, e=0.2 \& A=0.01$

\begin{tabular}{|l|l|l|l|l|l|l|l|}
\hline$a$ & $\rho L_{2}$ & $L_{2}$ & $\Omega_{\xi \xi}^{* 0}$ & $\Omega_{\eta \eta}^{* 0}$ & $\Omega_{\xi \xi}^{* 0} \Omega_{\eta \eta}^{* 0}$ & $\lambda_{1,2}$ & $\lambda_{3,4}$ \\
\hline 0.95 & 0.266817 & 0.694668 & 13.7206 & -4.4933 & -61.6504 & \pm 3.2999 & $\pm 2.3794 i$ \\
\hline 0.85 & 0.280115 & 0.681370 & 10.125 & -2.9932 & -30.3061 & \pm 2.6999 & $\pm 2.0390 i$ \\
\hline 0.75 & 0.300206 & 0.661279 & 7.2845 & -1.7985 & -13.1011 & \pm 2.1067 & $\pm 1.7181 i$ \\
\hline 0.65 & 0.330964 & 0.630521 & 5.2698 & -0.9393 & -4.9499 & \pm 1.5480 & $\pm 1.4373 i$ \\
\hline 0.55 & 0.376380 & 0.585105 & 4.0559 & -0.4094 & -1.6603 & \pm 1.0601 & $\pm 1.2154 i$ \\
\hline 0.45 & 0.437741 & 0.523744 & 3.4487 & -0.1327 & -0.4577 & \pm 0.6450 & $\pm 1.0489 i$ \\
\hline 0.35 & 0.513303 & 0.448182 & 3.1854 & -0.0003 & -0.4577 & \pm 0.0363 & $\pm 0.9035 i$ \\
\hline
\end{tabular}

Table 10. Effect of triaxiality on $L_{3}$ of CEN X-4 for $A=0.01, e=0.2 \& a=0.8$

\begin{tabular}{|l|l|l|l|l|l|l|l|l|}
\hline$\sigma_{1}$ & $\sigma_{2}$ & $\rho L_{3}$ & $L_{3}$ & $\Omega_{\xi \xi}^{* 0}$ & $\Omega_{\eta \eta}^{* 0}$ & $\Omega_{\xi \xi}^{* 0} \Omega_{\eta \eta}^{* 0}$ & $\lambda_{1,2}$ & $\lambda_{3,4}$ \\
\hline 0.01 & 0.0075 & 0.173749 & -0.86477 & 3.1517 & -0.0430 & -0.1354 & \pm 0.3637 & $\pm 1.0117 i$ \\
\hline 0.02 & 0.0150 & 0.178491 & -0.86002 & 3.1534 & -0.0432 & -0.1363 & \pm 0.3650 & $\pm 1.0115 i$ \\
\hline 0.03 & 0.0225 & 0.183125 & -0.85539 & 3.1550 & -0.0435 & -0.1373 & \pm 0.3663 & $\pm 1.0113 i$ \\
\hline 0.04 & 0.0333 & 0.186470 & -0.85205 & 3.1469 & -0.0437 & -0.1376 & \pm 0.3654 & $\pm 1.0150 i$ \\
\hline
\end{tabular}

Table 11. Effect of oblateness on $L_{3}$ of CEN X-4 for $\sigma_{1}=0.01, \sigma_{1}=0.0075, e=0.2 \& a=0.8$

\begin{tabular}{|l|l|l|l|l|l|l|l|}
\hline$A$ & $\rho L_{3}$ & $L_{3}$ & $\Omega_{\xi \xi}^{* 0}$ & $\Omega_{\eta \eta}^{* 0}$ & $\Omega_{\xi \xi}^{* 0} \Omega_{\eta \eta}^{* 0}$ & $\lambda_{1,2}$ & $\lambda_{3,4}$ \\
\hline 0.0001 & 0.175817 & -0.86270 & 3.1069 & -0.0430 & -0.1336 & \pm 0.3547 & $\pm 1.0305 i$ \\
\hline 0.001 & 0.175624 & -0.86289 & 3.1111 & -0.0430 & -0.1337 & \pm 0.3555 & $\pm 1.0287 i$ \\
\hline 0.01 & 0.173749 & -0.86477 & 3.1517 & -0.0429 & -0.1354 & \pm 0.3637 & $\pm 1.0117 i$ \\
\hline 0.1 & 0.159358 & -0.87916 & 3.4764 & -0.0428 & -0.1489 & \pm 0.4420 & $\pm 0.8728 i$ \\
\hline
\end{tabular}

Table 12. Effect of eccentricity on $L_{3}$ of CEN X-4 for $\sigma_{1}=0.01, \sigma_{2}=0.0075, a=0.8 \& A=0.1$

\begin{tabular}{|r|l|l|l|l|l|l|l|}
\hline \multicolumn{1}{|l|}{$e$} & $\rho L_{3}$ & $L_{3}$ & $\Omega_{\xi \xi}^{* 0}$ & $\Omega_{\eta \eta}^{* 0}$ & $\Omega_{\xi \xi}^{* 0} \Omega_{\eta \eta}^{* 0}$ & $\lambda_{1,2}$ & $\lambda_{3,4}$ \\
\hline 0.99 & 0.331701 & -0.70681 & 5.3882 & -0.0814 & -0.4384 & \pm 1.2584 & $\pm 0.5262 i$ \\
\hline 0.9 & 0.311435 & -0.72708 & 5.0390 & -0.0742 & -0.3738 & \pm 1.1230 & $\pm 0.5444 i$ \\
\hline 0.8 & 0.287995 & -0.75052 & 4.6915 & -0.0671 & -0.3148 & \pm 0.9769 & $\pm 0.5744 i$ \\
\hline 0.7 & 0.263891 & -0.77462 & 4.3863 & -0.0609 & -0.2673 & \pm 0.8394 & $\pm 0.6159 i$ \\
\hline 0.6 & 0.239624 & -0.79889 & 4.1226 & -0.0557 & -0.2294 & \pm 0.7167 & $\pm 0.6684 i$ \\
\hline 0.5 & 0.215932 & -0.82258 & 3.9001 & -0.0512 & -0.1997 & \pm 0.6146 & $\pm 0.7272 i$ \\
\hline 0.4 & 0.193814 & -0.84470 & 3.7183 & -0.0476 & -0.1770 & \pm 0.5359 & $\pm 0.7851 i$ \\
\hline 0.3 & 0.174504 & -0.86401 & 3.5772 & -0.0448 & -0.1603 & \pm 0.4794 & $\pm 0.8351 i$ \\
\hline
\end{tabular}


Table 13. Effect of semi-major axis on $L_{3}$ of CEN X-4 for $\sigma_{1}=0.03, \sigma_{2}=0.0225, e=0.2 \& A=0.01$

\begin{tabular}{|l|l|l|l|l|l|l|l|}
\hline$a$ & $\rho L_{3}$ & $L_{3}$ & $\Omega_{\xi \xi}^{* 0}$ & $\Omega_{\eta \eta}^{* 0}$ & $\Omega_{\xi \xi}^{* 0} \Omega_{\eta \eta}^{* 0}$ & $\lambda_{1,2}$ & $\lambda_{3,4}$ \\
\hline 0.95 & 0.081758 & -0.95676 & 3.1213 & -0.0379 & -0.1183 & \pm 0.3389 & $\pm 1.0152 i$ \\
\hline 0.85 & 0.148778 & -0.88974 & 3.1433 & -0.0415 & -0.1303 & \pm 0.3565 & $\pm 1.0125 i$ \\
\hline 0.75 & 0.218096 & -0.82042 & 3.1675 & -0.0458 & -0.1450 & \pm 0.3770 & $\pm 1.0101 i$ \\
\hline 0.65 & 0.290159 & -0.74836 & 3.1962 & -0.0512 & -0.1635 & \pm 0.4012 & $\pm 1.0080 i$ \\
\hline 0.55 & 0.365570 & -0.67295 & 3.2320 & -0.0581 & -0.1877 & \pm 0.4308 & $\pm 1.0058 i$ \\
\hline 0.45 & 0.445184 & -0.59333 & 3.2801 & -0.0674 & -0.2210 & \pm 0.4685 & $\pm 1.0034 i$ \\
\hline 0.35 & 0.530293 & -0.50822 & 3.3511 & -0.0807 & -0.2705 & \pm 0.5201 & $\pm 1.0001 i$ \\
\hline
\end{tabular}

Table 14. Effect of triaxiality on $L_{1}$ of PSR $1903+0327$ for $A=0.01, e=0.44 \& \quad a=0.85$

\begin{tabular}{|l|l|l|l|l|l|l|l|l|}
\hline$\sigma_{1}$ & $\sigma_{2}$ & $\rho L_{1}$ & $L_{1}$ & $\Omega_{\xi \xi}^{* 0}$ & $\Omega_{\eta \eta}^{* 0}$ & $\Omega_{\xi \xi}^{* 0} \Omega_{\eta \eta}^{* 0}$ & $\lambda_{1,2}$ & $\lambda_{3,4}$ \\
\hline 0.01 & 0.0075 & 0.529847 & 1.132735 & 4.9143 & -0.8693 & -4.2719 & \pm 1.4455 & $\pm 1.4298 i$ \\
\hline 0.02 & 0.0150 & 0.538001 & 1.140889 & 5.0752 & -0.8971 & -4.5531 & \pm 1.4915 & $\pm 1.4306 i$ \\
\hline 0.03 & 0.0225 & 0.545186 & 1.148074 & 5.2119 & -0.9208 & -4.7993 & \pm 1.5301 & $\pm 1.4318 i$ \\
\hline 0.04 & 0.0333 & 0.549976 & 1.152864 & 5.3751 & -0.9553 & -5.1346 & \pm 1.5766 & $\pm 1.4373 i$ \\
\hline
\end{tabular}

Table 15. Effect of oblateness on $L_{1}$ of PSR $1903+0327$ for $\sigma_{1}=0.01, \sigma_{1}=0.0075, e=0.44 \& a=0.85$

\begin{tabular}{|l|l|l|l|l|l|l|l|}
\hline$A$ & $\rho L_{1}$ & $L_{1}$ & $\Omega_{\xi \xi}^{* 0}$ & $\Omega_{\eta \eta}^{* 0}$ & $\Omega_{\xi \xi}^{* 0} \Omega_{\eta \eta}^{* 0}$ & $\lambda_{1,2}$ & $\lambda_{3,4}$ \\
\hline 0.0001 & 0.532153 & 1.135041 & 4.9032 & -0.8650 & -4.2414 & \pm 1.4417 & $\pm 1.4285 i$ \\
\hline 0.001 & 0.531942 & 1.134830 & 4.9042 & -0.8654 & -4.2442 & \pm 1.4421 & $\pm 1.4286 i$ \\
\hline 0.01 & 0.529847 & 1.132735 & 4.9143 & -0.8693 & -4.2719 & \pm 1.4455 & $\pm 1.4298 i$ \\
\hline 0.1 & 0.510474 & 1.113362 & 5.0120 & -0.9060 & -4.5409 & \pm 1.4780 & $\pm 1.4417 i$ \\
\hline
\end{tabular}

Table 16. Effect of eccentricity on $L_{1}$ of PSR $1903+0327$ for $\sigma_{1}=0.01, \sigma_{2}=0.0075, a=0.8 \& A=0.01$

\begin{tabular}{|r|l|l|l|l|l|l|l|}
\hline \multicolumn{1}{l|}{$e$} & $\rho L_{1}$ & $L_{1}$ & $\Omega_{\xi \xi}^{* 0}$ & $\Omega_{\eta \eta}^{* 0}$ & $\Omega_{\xi \xi}^{* 0} \Omega_{\eta \eta}^{* 0}$ & $\lambda_{1,2}$ & $\lambda_{3,4}$ \\
\hline 0.99 & 0.399139 & 1.002027 & 7.9769 & -1.7271 & -13.7765 & \pm 2.2368 & $\pm 1.6594 i$ \\
\hline 0.9 & 0.416178 & 1.019066 & 7.3069 & -1.5388 & -11.2440 & \pm 2.0861 & $\pm 1.6074 i$ \\
\hline 0.8 & 0.436093 & 1.038981 & 6.6528 & -1.3565 & -9.0242 & \pm 1.9291 & $\pm 1.5572 i$ \\
\hline 0.7 & 0.456788 & 1.059676 & 6.0890 & -1.2004 & -7.3095 & \pm 1.7844 & $\pm 1.5151 i$ \\
\hline 0.6 & 0.477829 & 1.080717 & 5.6105 & -1.0690 & -5.9977 & \pm 1.6537 & $\pm 1.4809 i$ \\
\hline 0.5 & 0.498557 & 1.101445 & 5.2131 & -0.9606 & -5.0077 & \pm 1.5387 & $\pm 1.4543 i$ \\
\hline 0.4 & 0.518061 & 1.120949 & 4.8931 & -0.8738 & -4.2755 & \pm 1.4413 & $\pm 1.4346 i$ \\
\hline 0.3 & 0.535201 & 1.138089 & 4.6473 & -0.8075 & -3.7525 & \pm 1.3634 & $\pm 1.4209 i$ \\
\hline
\end{tabular}

Table 17. Effect of semi-major axis on $L_{1}$ of PSR $1903+0327$ for $\sigma_{1}=0.03, \sigma_{2}=0.0225, e=0.44 \& A=0.01$

\begin{tabular}{|l|l|l|l|l|l|l|l|}
\hline$a$ & $\rho L_{1}$ & $L_{1}$ & $\Omega_{\xi \xi}^{* 0}$ & $\Omega_{\eta \eta}^{* 0}$ & $\Omega_{\xi \xi}^{* 0} \Omega_{\eta \eta}^{* 0}$ & $\lambda_{1,2}$ & $\lambda_{3,4}$ \\
\hline 0.95 & 0.569383 & 1.172271 & 5.5293 & -0.9973 & -5.5145 & \pm 1.6215 & $\pm 1.9518 i$ \\
\hline 0.85 & 0.545186 & 1.148074 & 5.2119 & -0.9208 & -4.7993 & \pm 1.5301 & $\pm 2.1042 i$ \\
\hline 0.75 & 0.519392 & 1.122280 & 4.8851 & -0.8391 & -4.0991 & \pm 1.4310 & $\pm 2.2744 i$ \\
\hline 0.65 & 0.491664 & 1.094552 & 4.5466 & -0.7510 & -3.4145 & \pm 1.3223 & $\pm 2.5944 i$ \\
\hline 0.55 & 0.461524 & 1.064412 & 4.1932 & -0.6550 & -2.7464 & \pm 1.2010 & $\pm 2.6769 i$ \\
\hline 0.45 & 0.428260 & 1.031148 & 3.8199 & -0.5486 & -2.0957 & \pm 1.0623 & $\pm 2.9206 i$ \\
\hline 0.35 & 0.390708 & 0.993596 & 3.4185 & -0.4283 & -1.4643 & \pm 0.8979 & $\pm 3.2111 i$ \\
\hline
\end{tabular}




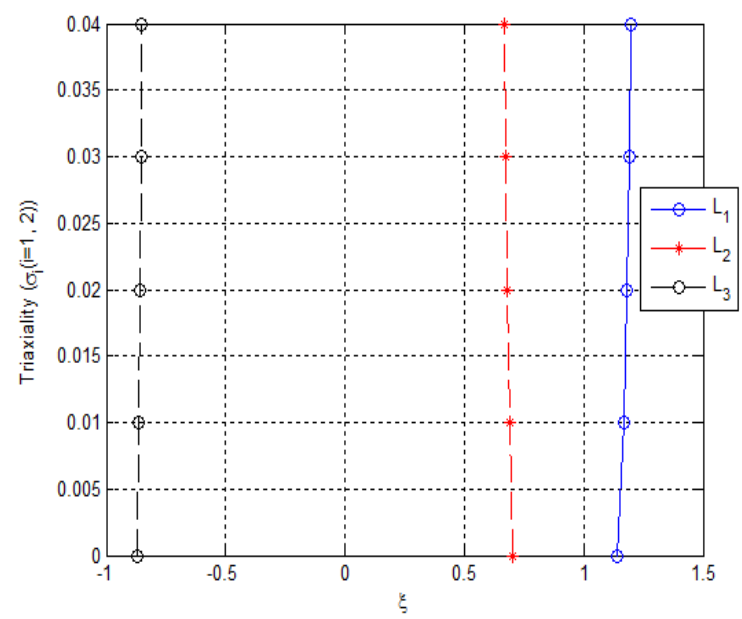

(a)

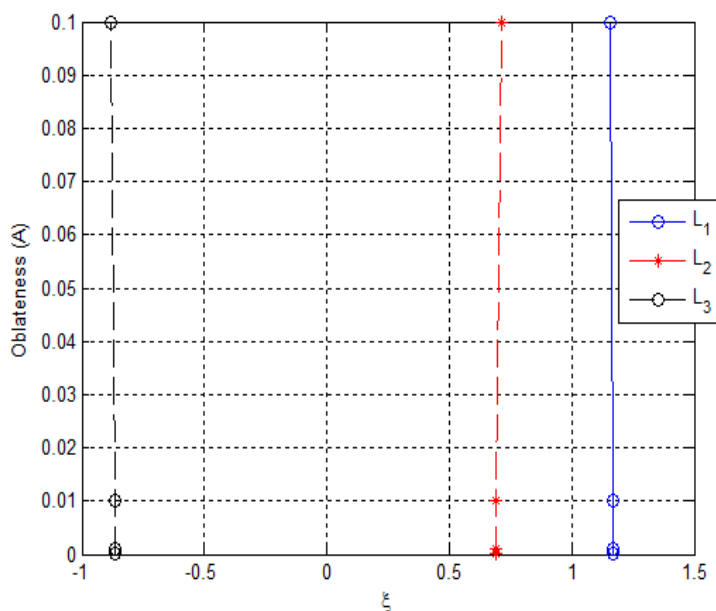

(b)

Figure 5. Effects of triaxiality (a) and oblateness (b) on the collinear libration points $L_{i}(i=1,2,3)$ of CEN X-4

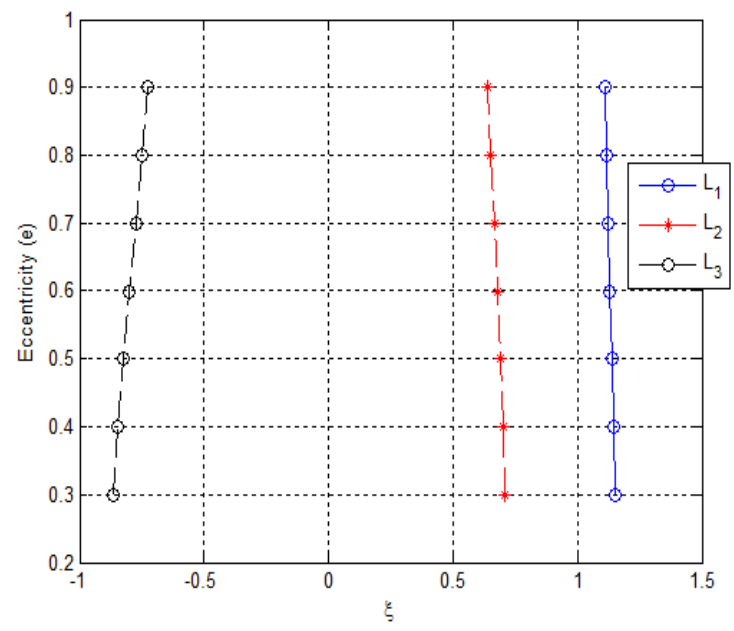

(a)

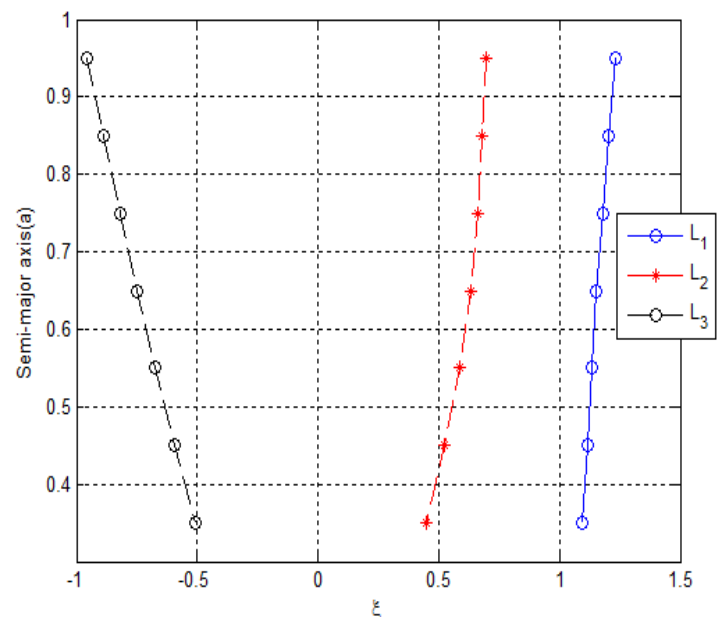

(b)

Figure 6. Effects of eccentricity (a) and semi-major axis (b) on the collinear libration points $L_{i}(i=1,2,3)$ of CEN X-4

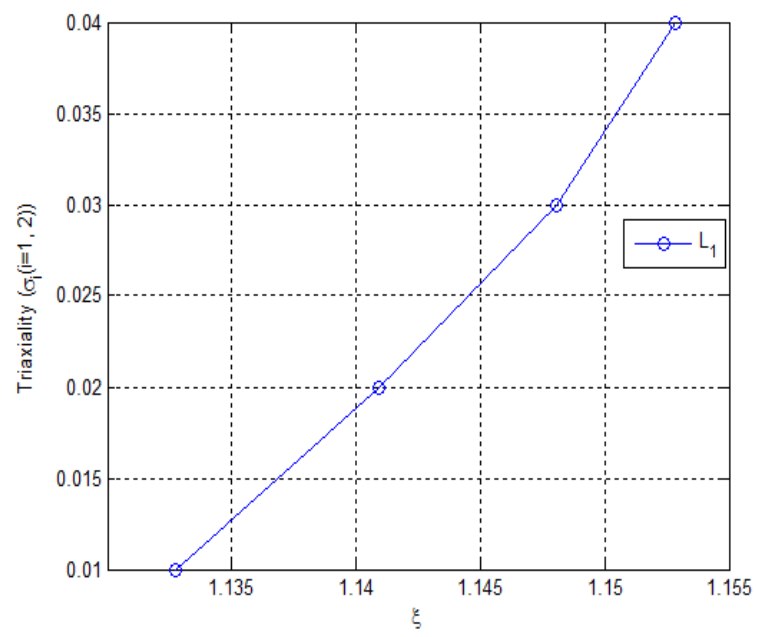

(a)

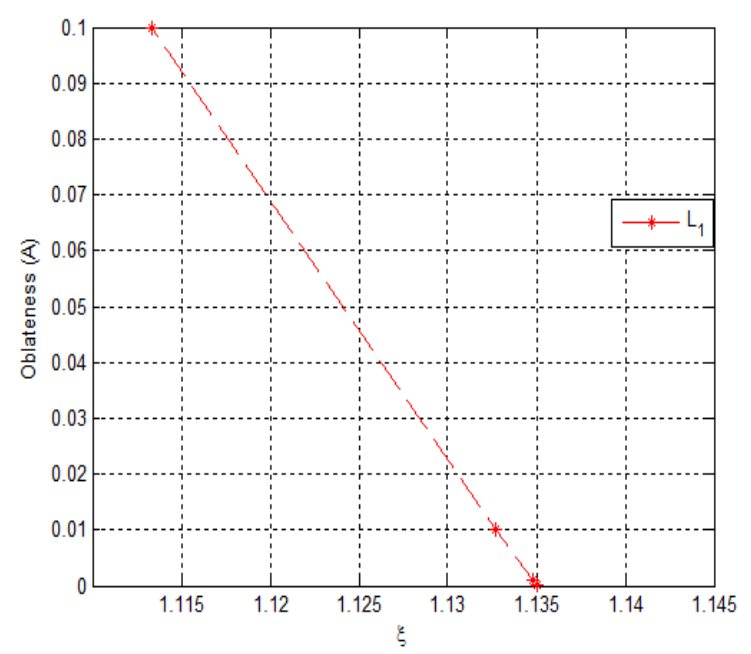

(b)

Figure 7. Effects of triaxiality (a) and oblateness (b) on the collinear libration point $L_{1}$ of PSR $1903+0327$ 


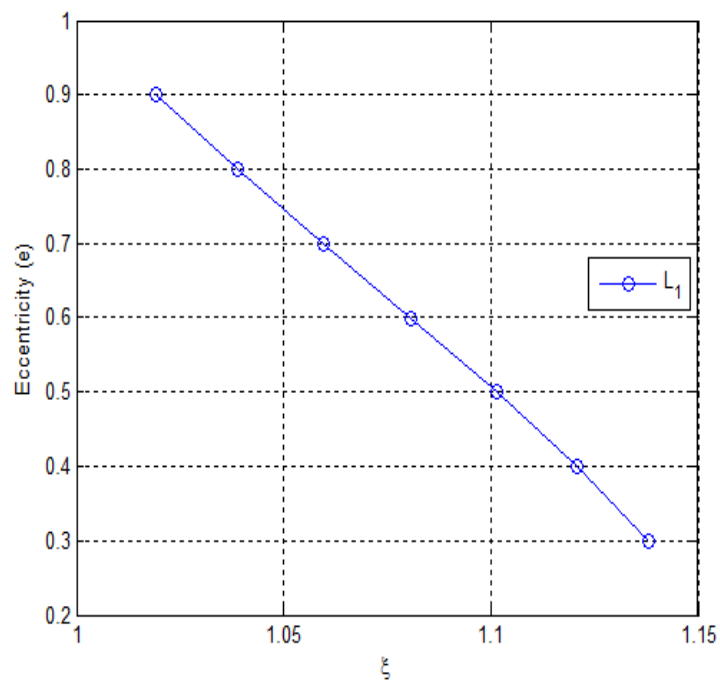

(a)

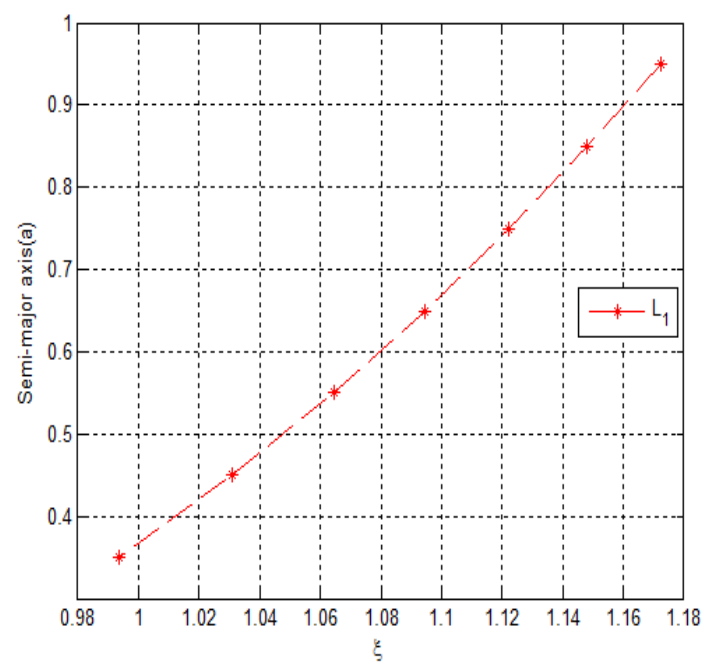

(b)

Figure 8. Effects of eccentricity (a) and semi-major axis (b) on the collinear libration point $L_{1}$ of PSR $1903+0327$

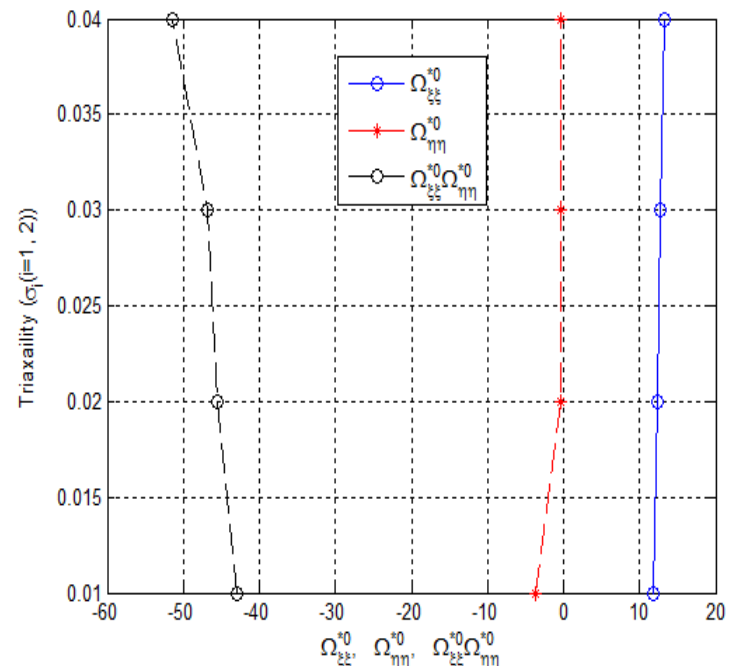

(a)

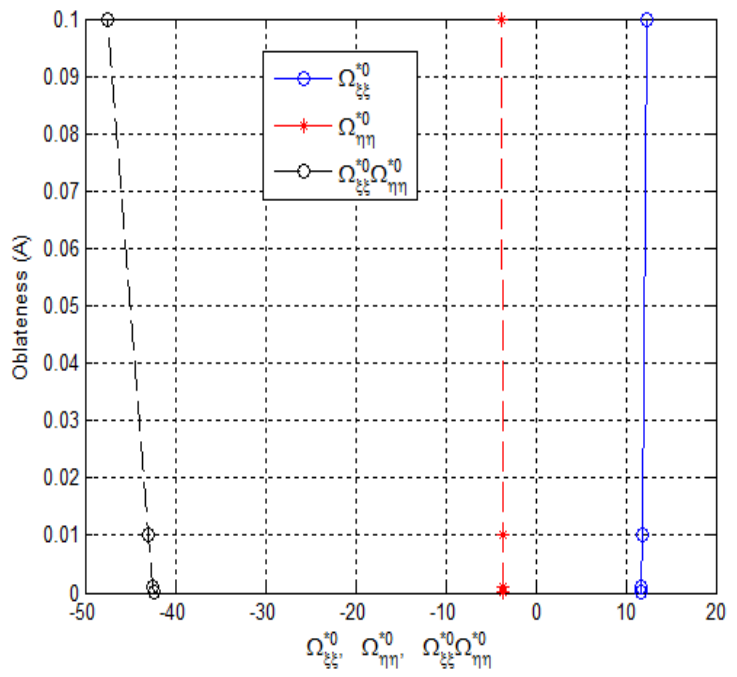

(b)

Figure 9. Effects of triaxiality (a) and oblateness (b) on the collinear libration point $L_{1}$ of CEN X-4

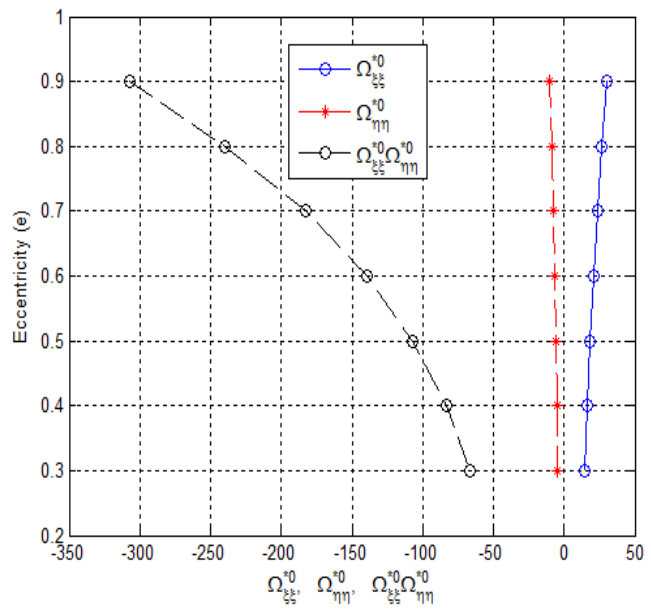

(a)

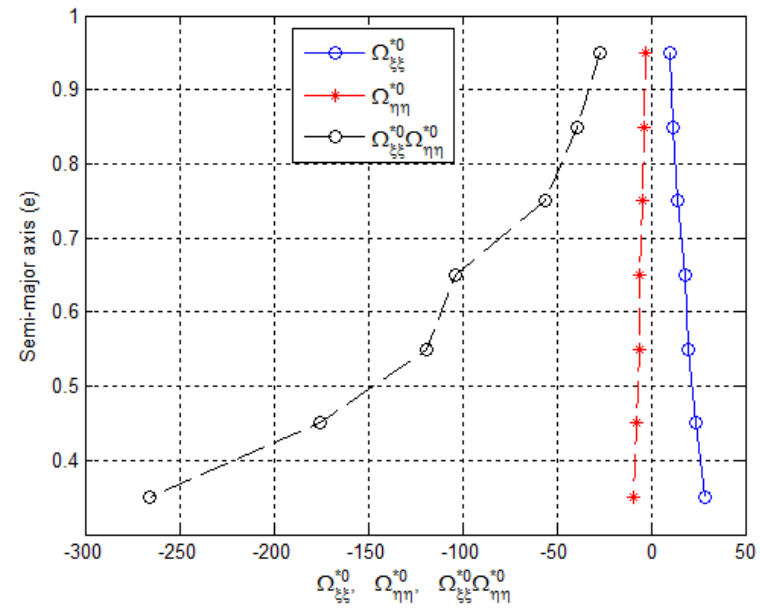

(b)

Figure 10. Effects of eccentricity (a) and semi-major axis (b) on the collinear libration point $L_{1}$ of 


\section{Discussion}

The positions and linear stability of the collinear libration points of the ER3BP under the effects of oblateness of the primary, the triaxiality and radiation of the secondary have been investigated. A numerical investigation of these effects on the positions of the collinear libration point $L_{1}$ of CEN X-4 and PSR J1903+0327 (Tables 2-9, Tables 14-17 and Figures 5-8) show that for both binaries, increase in triaxiality and eccentricity cause a shift away from the smaller primary while semi-major axis and oblateness tend to generate a shift towards the smaller primary (Figures 5-8). While increase in the triaxiality and eccentricity (Tables 6, 8, 10 and 12 and Figures 5a, and 6a) causes a shift towards the bigger primary; increase in oblateness and semi-major (Tables 7, 9, 11 and 13 and Figures $5 \mathrm{~b}$ and $6 \mathrm{~b}$ ) results in a shift away from the bigger primary on $L_{2,3}$.

The results of this study confirm the results obtained by [13, 31, 33, 34] when some of the parameters in both works are relaxed. Also, our result agree with [25] in the absence of relativistic parameter and triaxiality of the bigger primary $\left(c=\sigma_{1}=\sigma_{2}=0\right)$ in their work and as well, when relaxing ours to the classical case.

It is observed numerically and graphically that, the perturbing forces under consideration are unable to change the stability behaviour of the collinear libration points $\left(L_{i}(i=1,2,3)\right)$ due to the appearance of the real roots of the characteristic equation (equation (31)) (see Tables 2-17). This means that, the system is unbounded hence the collinear libration points are unstable in the Lyapunov sense. Graphically, has been highlighted the instability of the collinear libration point $L_{1}$ of CEN X-4. This validates [13, 25, 33, 34].

The stability of the system is significant for studying the trapping of a test particle in the vicinity CEN X-4 and PSR J1903+0327. It can also be useful for studying Trojan asteroids in the planetary system.

\section{References}

[1] V. Szebehely, Theory of orbits. The restricted problem of three bodies. Academic Press, New York, 1967.

[2] Lagrange, Collected Works Paris, VI, 229, 1772.

[3] G.D. Birkhoff, Dynamical system, American Mathematical Society, New York. 9 (1927).

[4] A.D. Bruno, The restricted 3-Body Problem. Plane periodic orbits, Walter de Gruyter, 1994.

[5] S.V. Ershkov, Stability of the moons orbits in solar system in the restricted three-body problem, Advances in Astronomy. 2015 (2015).

[6] V.V. Radzievskii, The restricted three-body problem including light pressure, Astron. J. 27 (1950) 250-256.

[7] V. Kumar, R.K. Choudhry, Nonlinear stability of the triangular libration points for the photogravitational elliptic restricted problem of three bodies, Celestial Mechanics and Dynamical Astronomy. 48(4) (1990) 299-317.

[8] M.K. Ammar, The effect of solar radiation pressure on the Lagrangian points in the elliptic restricted three-body problem, Astrophys. Space Sci. 313(4) (2008) 393-408.

[9] S. Kumar, B. Ishwar, Solutions of generalized photogravitational elliptic restricted three-body problem, AIP conference proceedings. 1146 (2009) 456.

[10] A. Narayan, C. Ramesh, Effects of photogravitational and oblantensss on the triangular Lagrangian points in the elliptical restricted three body problem, International Journal of Pure and Applied Mathematics. 68(2) (2011) 201-224. 
[11] J. Singh, A. Umar, Motion in the photogravitational elliptic restricted three-body problem under an oblate primary, The Astronomical Journal. 143(5) (2012) 109.

[12] A. Narayan, N. Singh, Stability of triangular Lagrangian points in the elliptic R3BP under radiating binary system, Astrophys. Space Sci. 353(2) (2014) 457-464.

[13] J. Singh, A. Umar, Collinear equilibrium points in the elliptic R3BP with oblateness and radiation, Advances in Space Research. 52 (2013) 1489-1496.

[14] J. Singh, A. Umar, Application of binary pulsars to axisymmetric bodies in the elliptic R3BP, Astrophys. Space Sci. 348 (2013) 393-402,.

[15] E.I. Abouelmagd, The effect of photogravitational force and oblateness in the perturbed restricted three-body problem, Astrophys. Space Sci. 346(1) (2013) 51-69.

[16] J.P. Rozelot, C. Damiani, History of solar oblateness measurements and interpretation, European Phys. J. H. 36(3) (2011) 407-436.

[17] K. Boshkayev, H. Quevedo, R. Ruffini, Gravitational field of compact objects in general relativity, Physical Review D. 86 (2012) Article ID: 064043.

[18] G.G. Arutyunyan, D.M. Sedrakyan, E.V. Chubaryan, Rotating white dwarfs in the general relativity theory, Astophys. Space Sci. 7(3) (1971) 274.

[19] J.S. Heyl, Gravitational radiation from strongly magnetized white dwarfs, Monthly Notices of the Royal Astronomical Society. 317(2) (2000) 310-314.

[20] L. Iorio, Dynamical determination of the quadrupole mass moment of a white dwarf, Astrophys. Space Sci. 310(1) (2007) 73-76.

[21] L. Iorio, Dynamical constraints on some orbital and physical properties of the WD0137349A/B binary system, Astrophys. Space Sci. 312(3) (2007) 337-341.

[22] J.D. Anderson et al., Shape, mean radius, gravity field, and interior structure of Callisto, Icarus. 153(1) (2001) 157-161.

[23] J. Yan et al., Comparison analyses on the $150 \times 150$ lunar gravity field models by gravity/topography admittance, correlation and precision orbit determination, Advances in Space Research. 52(3) (2013) 512-520.

[24] A. Umar, A.A. Hussain, Motion in the ER3BP with an oblate primary and triaxial stellar companion, Astophys. Space Sci. 361(10) (2016) 344.

[25] B. Nakone, A.A. Hussain, Collinear equilibrium points in the relativistic R3BP when the bigger primary is a triaxial rigid body, International Frontier Science Letters. 11 (2017) 45-56.

[26] A. Narayan, K.K. Pandey, S.K. Sharivastava, Effects of radiation and triaxiality of triangular equilibrium points in elliptical restricted three-body problem, International Journal of Advanced Astronomy. 3(2) (2015) 97-106.

[27] H. Peng, X. Bai, S. Xu, Continuation of periodic orbits in the Sun-Mercury elliptic restricted three-body problem, Commun. Nonlinear Sci. Numer. Simulat. 47 (2017) 1-15.

[28] E.I. Abouelmagd, S.M. El- Shaboury, Periodic orbits under combined effects of oblateness and radiation in the restricted problem of three bodies, Astrophys. Space Sci. 341(2) (2012) 331-341.

[29] N.I. Vishnu Namboori, D. Sudheer Reedy, R.K. Sharma, Effect of oblateness and radiation pressure on angular frequencies at collinear points, Astrophys. Space Sci. 318(3-4) (2008) 161-168.

[30] K. Yamada, H. Asada, Uniqueness of collinear solutions for the relativistic three-body problem, Physical Review D. 83(2) (2011) 024040. 
[31] F. Alzahrani et. al., On the libration collinear points in the restricted three-body problem, De Gruyter Open. 15 (2017) 58-67.

[32] S.M. El Shaboury, A. Mostafa, The singly averaged elliptical restricted three-body problem, Astrophys. Space Sci. 348(2) (2013) 385-391.

[33] S.K. Sahoo, B. Ishwar, Stability of collinear equilibrium points in the generalized photogravitational elliptic restricted three-bodyproblem, Bull. Astron. Soc. India. 28 (2000) 579.

[34] S. Kumar, B. Ishwar, Locations of collinear equilibrium points in the generalized elliptic restricted three-body problem, International Journal of Engineering, Science and Technology. 3 (2011) 157-162.

[35] A.Narayan, K.K. Pandey, S.K. Sharivastava, Characteristics exponents of the triangular solution in the elliptic restricted three-boy problem under radiating and triaxial primaries, International Journal of Advanced Astronomy. 5(1) (2017) 12-18.

[36] J. Singh, A. Umar, On motion around the collinear libration points in the elliptic restricted three-body problem with a bigger triaxial primary, New Astronomy. 29 (2014) 36-41.

[37] A.S. Zimovshchikov, V.N. Tkhai, Stability diagrams for a heterogeneous ensemble of particles at the collinear libration points of the photogravitational three-body problem, J. App. Math. Mech. 74(2) (2010) 158-163.

[38] J. Singh, A. Umar, The collinear libration points in the elliptic R3BP with a triaxial primary and an oblate secondary, International Journal of Astronomy and Astrophysics. 4(2014)391-398. 